\title{
Effects of the Nearest-Neighbour Coulomb Interactions on the Ground State of the Periodic Anderson Model
}

\author{
Sushil Lamba, R. Kishore \\ Laboratório Associado de Sensores e Materiais, Instituto Nacional de Pesquisas Espaciais, 12225 Saõ José dos Campos, São \\ Paulo, Brazil \\ S. K. Joshi \\ National Physical Laboratory, Dr. K. S. Krishnan Marg, New Delhi 110 012, India and Jawaharlal Nehru Center for \\ Advanced Scientific Research, IISc Campus, Bangalore 560 012, India.
}

(August 4, 1997)

The magnetic and non-magnetic ground states of the periodic Anderson model with Coulomb interaction between $f$ electrons on the nearest-neighbour $(\mathrm{NN})$ sites are investigated using a variational method, which gives exact calculation of the expectation values in the limit of infinite dimensions. It is shown that for a critical value of NN Coulomb interactions the magnetic ground state of the periodic Anderson model in the Kondo regime is unstable. Factors in terms of the physical processes responsible for instability of the magnetic ground state are also discussed. Our study indicates the importance of the NN Coulomb interactions for correlated two band models.

PACS numbers: 71.27.+a, 75.20.Hr, 75.30.Mb

Over the past decade lot of effort has been devoted to the theoretical understanding of the ground state properties of the heavy-fermion systems. One of the intriguing experimentally observed phenomena in the heavyfermion materials is the variety of magnetic and nonmagnetic ground states observed in these materials 6 . Most of the theoretical investigations of the magnetic properties are done on the basis of the periodic Anderson model(PAM) assuming that this model contains the essential physics of these materials. Theoretical approaches based on the slave-boson techniques 9 are biased towards a paramagnetic ground state while variational approaches based on the Gutzwiller method are biased towards a magnetic state. These two approaches are equivalent in the limit of large orbital degeneracy. Recently, Reynolds et. al 10 , studied the magnetic properties of the orbitally non-degenerate periodic Anderson model using Kotliar and Ruckenstein slave-boson(KRSB) formulation of the Gutzwiller method. In this approach the Gutzwiller approximation is reproduced at the saddle point for $T=0$. They found that a magnetic instability exists in the entire Kondo regime and therefore, the Gutzwiller approximation is too biased towards the magnetic ground state. The experimental evidence points to the gross inadequacy of the existing approaches to describe the magnetic behaviour of heavy fermions.

In addition to the on-site Coulomb interaction in the $f$ band, the other most important interactions which may affect the stability of the magnetic ground state of the
PAM are the on-site Coulomb interaction in conduction band and the NN Coulomb interaction in the $f$ band. The influence of the on-site Coulomb interaction in the conduction band was recently considered by Itai and Fazekas 11 using the Gutzwiller variational method. They found that this interaction reduces the Kondo scale. The reduced Kondo scale implies that the transitions of electrons from the $f$-band to the conduction band and vice versa, are further restricted by the presence of the Coulomb interactions in the conduction band. This would lead to further enhancement of the magnetic ordering of the ground state of the periodic Anderson model. Consequently, the ground state of the periodic Anderson model with the on-site Coulomb interaction in the conduction band would be magnetic in the entire Kondo regime. In the presence of the NN Coulomb interaction in the $f$-band all the configurations, having electrons on the NN sites are energetically unfavourable and the following physical processes would be operatin (i) $f$-electrons can avoid NN Coulomb repulsion by occupying next to nearest neighbour sites. This process is expected to be important only when sufficient number of vacant sites are available. (ii) Electrons from the $f$-band may go to the Fermi level whereby take advantage of the hybridization interaction to delocalize. (iii) Spin-flip process in the $f$-band through hybridization interaction would also lead to energy gain. All these processes would affect the magnetic ordering of the ground state of the PAM. The purpose of this paper is to investigate the influence of the NN Coulomb interaction in the $f$-band, on the magnetic instability of the ground state of the PAM in the Kondo regime. To the best of our knowledge, this is the first study of the influence of the NN Coulomb interaction on the ground state properties of the PAM.

We consider the extended periodic Anderson model given by

$$
\begin{aligned}
H= & \sum_{k, \sigma} \epsilon_{k} d_{k \sigma}^{\dagger} d_{k \sigma}+\sum_{i, \sigma} E_{f} \hat{n}_{f i \sigma}+V \sum_{i, \sigma}\left(d_{i \sigma}^{\dagger} f_{i \sigma}+h . c\right)+ \\
& \frac{U}{2} \sum_{i, \sigma} n_{f i \sigma} n_{f i-\sigma}+G \sum_{\langle i j\rangle \sigma \sigma^{\prime}} n_{f i \sigma} n_{f j \sigma^{\prime}}
\end{aligned}
$$

where $n_{f i \sigma}=f_{i \sigma}^{\dagger} f_{i \sigma}, i$ and $j$ are site indices and $k$ are the wave vectors. The first four terms constitute the 
standard PAM and the last term in the Hamiltonian corresponds to the Coulomb interaction between $f$-electrons on the NN sites. $\sum_{\langle i j\rangle}$ in the last term denotes that the sum is taken over NN sites only. The total density of electrons $n=\left(\sum_{i \sigma} n_{f i \sigma}+n_{d i \sigma}\right) / N$, where $N$ is the total number of lattice sites, is taken to be $1<n<2$, so that there are enough electrons to fill atleast the $f$-levels, and the $d$-band filling is variable up to half filling.

To study the magnetic ground state we generalize the variational method previously used to investigate the paramagnetic regime of the PAM12 14. The generalizations are carried out by distinguishing the up and down spin electrons in the variational wave function. In the previous treatment for the paramagnetic regime of the PAM only the lower two spin-degenerate hybridized quasiparticle bands were considered, however, for the more general case of magnetism, it is required to take into consideration all four hybridized quasiparticle bands in the variational wave function.

To investigate magnetic ground state, we choose the variational wave function as:

$$
\left|\psi_{c}\right\rangle=\prod_{i} P_{i}\left|\psi_{u c}\right\rangle
$$

Where $\left|\psi_{u c}\right\rangle=\prod_{k, k^{\prime}, \sigma, \sigma^{\prime}}^{\prime} l_{k \sigma}^{\dagger} u_{k^{\prime} \sigma^{\prime}}^{\dagger}|0\rangle$ is the uncorrelated wave function. $\prod^{\prime}$ denotes the product over all occupied states. $u_{k \sigma}^{\dagger}$ and $l_{k \sigma}^{\dagger}$ create quasiparticles in the upper and lower hybridized bands respectively. $l_{k \sigma}^{\dagger}=$ $\alpha_{k \sigma} d_{k \sigma}^{\dagger}-\beta_{k \sigma} f_{k \sigma}^{\dagger}$ and $u_{k \sigma}^{\dagger}=\alpha_{k \sigma} f_{k \sigma}^{\dagger}+\beta_{k \sigma} d_{k \sigma}^{\dagger} \cdot \alpha_{k \sigma}$ and $\beta_{k \sigma}$ are variational functions, which denote the probability amplitude for conduction $(d)$ and $f$-electrons in the various quasiparticle bands. The quasiparticle creation operators $l_{k \sigma}^{\dagger}$ and $u_{k \sigma}^{\dagger}$ obey fermion commutation rule if $\alpha_{k \sigma}^{2}+\beta_{k \sigma}^{2}=1$. The variational functions $\alpha_{k \sigma}$ and $\beta_{k \sigma}$ differ from the choice which diagonalizes the Hamilto$\operatorname{nian}(\mathrm{Eq} .1$ ) in the absence of Coulomb interactions. Because the Coulomb interactions between the $f$-electrons can renormalize the hybridization interaction between $d$ and $f$-electron and thereby can also change the probability amplitudes. The correlation operator, $P_{i}$, is introduced to suppress those configurations in the uncorrelated state which are not energetically favourable in the presence of Coulomb interactions. The correlation operator 1214 is given by

$$
P_{i}=1+\sum_{\sigma} s_{\sigma} n_{f i \sigma}-\left[(1-d)+\sum_{\sigma} s_{\sigma}\right] n_{f i \uparrow} n_{f i \downarrow}
$$

The ground state energy per site of the trial wave func$\operatorname{tion}(\mathrm{Eq} .2)$ is given by $E_{g} / N=\left\langle\psi_{c}|H / N| \psi_{c}\right\rangle /\left\langle\psi_{c} \mid \psi_{c}\right\rangle$. The exact calculation of the ground state energy of the correlated wave function is not possible since the expectation values involve infinite product of operators and one needs to adopt some approximatescheme. In this paper we use the one-site approximation $12-14$ for calculation of various matrix elements appearing in the ground state energy per site of $\left|\psi_{c}\right\rangle$. The expectation values appearing in the ground state energy per site of $\left.\psi_{c}\right\rangle$, typically involve expectation values of the type $\left\langle\ldots n_{f i \sigma} n_{f j \sigma^{\prime}} . .\right\rangle_{u c}$. In the one-site approximation, such expectation values are approximated by

$$
\left\langle\ldots n_{f i \sigma} n_{f j \sigma^{\prime}} . .\right\rangle_{u c}=\langle\ldots\rangle_{u c}\left\langle n_{f i \sigma}\right\rangle_{u c}\left\langle n_{f j \sigma^{\prime}}\right\rangle_{u c}\langle\ldots\rangle_{u c}
$$

where $\langle\ldots\rangle_{u c}=\left\langle\psi_{u c}|\ldots| \psi_{u c}\right\rangle$. Such an approximation implies the collapse of all intersite diagrams in the position space [see Figure 1].

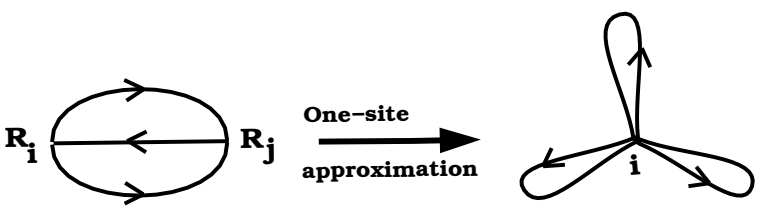

FIG. 1. The collapse of intersite diagrams in the one-site approximation.

The one-site approximation is expected to give exact calculation of the expectation values in the limit of infinite dimensions, since as dimension increases the contribution of the intersite diagrams decreases and vanishes altogether in the limit of infinite dimensions 15 .

Using the one-site approximation described above to calculate the expectation values appearing in the ground state energy of $\left|\psi_{c}\right\rangle$ and minimizing the energy functional with respect to the variational functions $\alpha_{k \uparrow}, \alpha_{k \downarrow}, \beta_{k \uparrow}$, $\beta_{k \downarrow}$ by imposing the constraint $\alpha_{k \sigma}^{2}+\beta_{k \sigma}^{2}=1$, the minimum of the ground-state energy per site is given by

$$
\begin{aligned}
\frac{E_{g}}{N}= & \frac{1}{N} \sum_{k \sigma}\left[\xi_{k \sigma}^{-}\left\langle l_{k \sigma}^{\dagger} l_{k \sigma}\right\rangle+\xi_{k \sigma}^{+}\left\langle u_{k \sigma}^{\dagger} u_{k \sigma}\right\rangle\right]+ \\
& \sum_{\sigma} \mu_{\sigma} n_{f \sigma}+U D+G \sum_{\sigma}\left(I_{\sigma}^{2}+I_{\sigma} I_{-\sigma}\right)
\end{aligned}
$$

where $\xi_{k \sigma}^{ \pm}$describes four hybridized quasiparticle bands

$$
\xi_{k \sigma}^{ \pm}=\frac{1}{2}\left[\left(\epsilon_{k}+\tilde{E}_{f \sigma}\right) \pm\left[\left(\epsilon_{k}-\tilde{E}_{f \sigma}\right)^{2}+4 \tilde{V}_{\sigma}^{2}\right]^{\frac{1}{2}}\right]
$$

and $\left\langle u_{k \sigma}^{\dagger} u_{k \sigma}\right\rangle_{u c}$ and $\left\langle l_{k \sigma}^{\dagger} l_{k \sigma}\right\rangle_{u c}$ correspond to the average occupation of the upper $\left(\xi_{k \sigma}^{+}\right)$and lower $\left(\xi_{k \sigma}^{-}\right)$quasiparticle bands. $\tilde{E}_{f \sigma}=E_{f}-\mu_{\sigma}$ is the renormalized $f$-level energy with the $f$-electron self energy $\mu_{\sigma}$ given by

$$
\begin{aligned}
\mu_{\sigma}= & -\frac{2}{N} \sum_{k \sigma^{\prime}} \tilde{V}_{\sigma^{\prime}} \frac{\partial \tilde{V}_{\sigma^{\prime}}}{\partial n_{f \sigma}}\left[\frac{\left\langle u_{k \sigma^{\prime}}^{\dagger} u_{k \sigma^{\prime}}\right\rangle_{u c}-\left\langle l_{k \sigma^{\prime}}^{\dagger} l_{k \sigma^{\prime}}\right\rangle_{u c}}{\sqrt{\left(\epsilon_{k}-\tilde{E}_{f \sigma^{\prime}}\right)^{2}+4 \tilde{V}_{\sigma^{\prime}}^{2}}}\right]- \\
& U \frac{\partial D}{\partial n_{f \sigma}}-G \frac{\partial \sum_{\sigma}\left(I_{\sigma}^{2}+I_{\sigma} I_{-\sigma}\right)}{\partial n_{f \sigma}} .
\end{aligned}
$$

$\tilde{V}_{\sigma}=V R_{\sigma}$ is the renormalized hybridization interaction and $R_{\sigma}$ is the renormalization factor. $R_{\sigma}$ and the average 
double occupancy, $D$ of the ground state $\left|\psi_{c}\right\rangle$ are given by

$$
\begin{gathered}
R_{\sigma}=\frac{\left(1-n_{f}\right)}{A}\left[\left(1-n_{f-\sigma}\right)\left(1+s_{\sigma}\right)+d n_{f-\sigma}\left(1+s_{-\sigma}\right)\right] \\
D=d^{2}\left(1-n_{f}\right) n_{f \uparrow} n_{f \downarrow} / A
\end{gathered}
$$

$I_{\sigma}=n_{f \sigma}\left[\left(1+s_{\sigma}^{2}+n_{f-\sigma}\left(d^{2}-\left(1+s_{\sigma}\right)^{2}\right)\right]\left(1-n_{f}\right) / A\right.$ with $A=\left(1-n_{f}\right)+\left(1-d^{2}\right) n_{f \uparrow} n_{f \downarrow}$. The density of $f$ electrons $n_{f \sigma}$ is given by

$$
n_{f \sigma}=\frac{1}{N} \sum_{k}\left[\beta_{k \sigma}^{2}\left\langle l_{k \sigma}^{\dagger} l_{k \sigma}\right\rangle_{u c}+\alpha_{k \sigma}^{2}\left\langle u_{k \sigma}^{\dagger} u_{k \sigma}\right\rangle_{u c}\right]
$$

The weight factors $\beta_{k \sigma}^{2}$ and $\alpha_{k \sigma}^{2}$ for $f$-electrons with spin $\sigma$ in the lower and upper quasiparticle bands respectively are given by

$$
\begin{gathered}
\alpha_{k \sigma}=\frac{-\left(\epsilon_{k}+\tilde{E}_{f \sigma}\right)+\left[\left(\epsilon_{k}-\tilde{E}_{f \sigma}\right)^{2}+4 \tilde{V}_{\sigma}^{2}\right]^{\frac{1}{2}}}{\sqrt{2}\left[\left(\epsilon_{k}-\tilde{E}_{f \sigma}\right)^{2}+4 \tilde{V}_{\sigma}^{2}\right]^{\frac{1}{4}}} \\
\beta_{k \sigma}=\frac{\left(\epsilon_{k}+\tilde{E}_{f \sigma}\right)+\left[\left(\epsilon_{k}-\tilde{E}_{f \sigma}\right)^{2}+4 \tilde{V}_{\sigma}^{2}\right]^{\frac{1}{2}}}{\sqrt{2}\left[\left(\epsilon_{k}-\tilde{E}_{f \sigma}\right)^{2}+4 \tilde{V}_{\sigma}^{2}\right]^{\frac{1}{4}}}
\end{gathered}
$$

The minimization of the ground state energy with respect to $d$ yield the following implicit equation for $d$.

$$
\begin{aligned}
U \frac{\partial D}{\partial d}= & -\frac{2}{N} \sum_{k \sigma^{\prime}} \tilde{V}_{\sigma^{\prime}} \frac{\partial \tilde{V}_{\sigma^{\prime}}}{\partial d}\left[\frac{\left\langle u_{k \sigma^{\prime}}^{\dagger} u_{k \sigma^{\prime}}\right\rangle_{u c}-\left\langle l_{k \sigma^{\prime}}^{\dagger} l_{k \sigma^{\prime}}\right\rangle_{u c}}{\sqrt{\left(\epsilon_{k}-\tilde{E}_{f \sigma^{\prime}}\right)^{2}+4 \tilde{V}_{\sigma^{\prime}}^{2}}}\right] \\
& -G \frac{\partial \sum_{\sigma}\left(I_{\sigma}^{2}+I_{\sigma} I_{-\sigma}\right)}{\partial d} .
\end{aligned}
$$

At zero temperatures, we can replace the distribution function for the lower and upper quasiparticle bands by unit step functions; $\left\langle l_{k \sigma}^{\dagger} l_{k \sigma}\right\rangle_{u c}=\Theta\left(-\xi_{k \sigma}^{-}+\nu\right)$ and $\left\langle u_{k \sigma}^{\dagger} u_{k \sigma}\right\rangle_{u c}=\Theta\left(-\xi_{k \sigma}^{+}+\nu\right)$. Here $\Theta$ is the unit step function, and $\nu$ is the Fermi level. $\nu$ is determined by fixing the density of total number of electrons per site, $n=\sum_{\sigma} n_{\sigma}$. At zero temperatures $n_{\sigma}$ is given by the following expression

$$
n_{\sigma}=\frac{1}{N} \sum_{k}\left[\Theta\left(-\xi_{k \sigma}^{-}+\nu\right)+\Theta\left(-\xi_{k \sigma}^{+}+\nu\right)\right] .
$$

Before embarking on the numerical calculations it would be instructive to compare our approach for the periodic Anderson mode wwith the KRSB reformulation of the Gutzwiller method 10 . We note that the ground-state energy of our variational wave function in the one-site approximation and the ground-state energy derived from the KRSB approach have different expressions for the effective hybridization interaction and the average double occupancy of the ground state. The average double occupancy in the one site approximation and the Gutzwiller approximation are given by $D$ and $d_{g}$ (say) respectively. If we scale $D \rightarrow d_{g}$ in the expression for the effective hybridization $\left(\tilde{V}_{\sigma}\right)$ in our approach, we find that it reduces to the corresponding expression for the effective hybridization in the KRSB method. This further implies that the $f$-electron self-energy $\left(\mu_{\sigma}\right)$, and the average occupation of the $f$-orbitals in both the approaches also become the same; thereby the KRSB ground-state energy functional and the one-site ground state energy functional are the same under the scaling of average double occupancy of the ground state. Furthermore, since both the approaches search for the minimum of the groundstate energy in the same physical parameter space, they must give the same results at the point of minimum. The equivalence of the two seemingly different variational methods is surprising. To understand this equivalence, we reanalyze the Gutzwiller variational wave function. The Gutzwiller wave function has a long history, dating back to the work of Gutzwiller in 1960's. The Gutzwiller wave function 16.17 is given by $\left|\psi_{g}\right\rangle=g^{\hat{D}}\left|\psi_{o}\right\rangle$. Recently, Gebhard 18.19 showed that it is more convenient to work with the following form for the Gutzwiller wave function.

$$
\left|\psi_{g k}\right\rangle=g^{\hat{K}}\left|\phi_{o}\right\rangle
$$

where $\left|\phi_{o}\right\rangle$ is an arbitrary normalized one-particle product wave function and $\hat{K}=\hat{D}-\sum_{i \sigma} \mu_{i \sigma} n_{f i \sigma}$, where $\mu_{i \sigma}$ are the explicit functions of $g$ and the local occupation of $f$-orbitals, $n_{f i \sigma}=\left\langle\phi_{o}\left|n_{f i \sigma}\right| \phi_{o}\right\rangle .\left|\psi_{o}\right\rangle$ and $\left|\phi_{o}\right\rangle$ are connected by $\left|\psi_{o}\right\rangle=g^{\sum_{i \sigma} \mu_{i \sigma} n_{f i \sigma}}\left|\phi_{o}\right\rangle$ For the magnetic case the correlator $g^{\hat{K}}$ can be written as $\prod_{i} Q_{i}$ with $Q_{i}=1+x n_{f i \uparrow} n_{f i \downarrow}-\sum_{\sigma} y_{\sigma} n_{f i \sigma}, x$ and $y_{\sigma}$ are variational parameters which depend on the average occupation of the $f$-orbitals. With the redefinition of the parameters $x$ and $y_{\sigma}$, the correlation operators $Q_{i}$ and $P_{i}$ (Eq. 3) are the same. Therefore, the GutzwillerGebhard correlator $g^{\hat{K}}$ in the Gutzwiller approximation and our correlator $\prod_{i} P_{i}$ in one-site approximation, describe the same physics. It is interesting to note that the Gutzwiller approximation gives the exact calculation of the matrix elements in the limit of infinite dimensions and gives identical results as obtained by the one-site approximation. Obviously, one-site approximation is much more physically transparent and operationally simpler than the Gutzwiller approximation.

Although our variational formalism is valid for arbitrary dimension and dispersion of conduction electron band, for simplicity we assume a conduction band with a constant density of states $\rho\left(\epsilon_{k}\right)=1 / 2 W$ lying in the energy interval $-W \leq \epsilon_{k} \leq W .2 W$ is the conduction electron band width. We have also taken infinite- $U$ limit, since at $U=\infty$ the ground state of the PAM is strongly magnetic with maximum value of total magnetization 10 . This is an ideal limit to investigate the instability of the magnetic ground state in the presence of the near- 
est neighbour Coulomb interactions. In our formalism this limit is affected by putting $d=0$ throughout i.e., by projecting out all the doubly occupied sites. The total magnetization, $m=\sum_{\sigma} \sigma n_{\sigma}$ for different values of nearest neighbour interaction $G$, the bare hybridization, $V$ and the total electron density, $n$, is calculated numerically by solving Eq. (6), Eq. (7), and Eq. (10) selfconsistently for $\mu_{\uparrow}, \mu_{\downarrow}, n_{f \uparrow}, n_{f \downarrow}$, and $\nu$. The numerical solution of the self-consistent equations have more than one solutions corresponding to strong magnetism, weak magnetism and paramagnetism. The relevant solution is one with the lowest ground state energy. In the numerical calculations we took the conduction electron bandwidth, $2 W=20 \mathrm{eV}$ and the $f$-level, $E_{f}=-1.5 \mathrm{eV}$ below the middle of the conduction band. We have taken this particular choice of parameter values for reasons of comparison with earlier work of Reynolds et a 10 in the absence of nearest neighbour Coulomb interaction between the $f$-electrons.

In Figure 2, we have plotted the total magnetization as a function of the NN Coulomb interaction $G$, for the total density of electrons, $n=1.95$ and $n=1.9$. For $G /\left|E_{f}\right|=0$, the ground state is strongly ferromagnetic with total magnetization, $m=0.96$ for $n=1.95$ and $m=.94$ for $n=1.9$. With increasing value of $G$ the magnetization decreases upto to a critical value of $G$, where we see a crossover from strong ferromagnetism to weak ferromagnetism with total magnetization $m=2-n$ and then from weak ferromagnetism to paramagnetism. Figure 3 shows the magnetic phase diagram of the extended periodic Anderson model.

In Figure 4 we have plotted the hybridized quasiparticle bands $\xi_{k \sigma}^{ \pm}$for strongly ferromagnetic $\left(G /\left|E_{f}\right|=0\right)$, weakly ferromagnetic $\left(G /\left|E_{f}\right|=2\right)$ and paramagnetic $\left(G /\left|E_{f}\right|=5.6\right)$ ground states. We find that due to the renormalization of the hybridization interaction and the $f$-electron energy there is a redistribution of the density of states and to accommodate the redistribution of density of states the Fermi level also moves to keep the total density of electrons fixed. For the strongly magnetic ground state the Fermi level lies in the lower down spin hybridized band $\left(\xi_{k \downarrow}^{-}\right)$and the upper up-spin hybridized band $\left(\xi_{k \uparrow}^{+}\right)$. The lower up-spin hybridized band $\left(\xi_{k \uparrow}^{-}\right)$is completely full. At a critical value of $G /\left|E_{f}\right|$ all the electrons in the upper hybridized up-spin band are transferred to the lower hybridized down-spin band. Then we see a crossover from strongly ferromagnetic to weakly ferromagnetic ground state. In the weakly ferromagnetic ground state, the lower hybridized up-spin band is completely full with total density of up-spin electrons, $n_{\uparrow}=1$, therefore the total magnetization, $m=n_{\uparrow}-\left(n-n_{\uparrow}\right)=2-n$. The total magnetization remains unchanged in the entire weak ferromagnetic regime till the fermi level also lies in the lower hybridized upspin band. To understand the magnitude of jump in the magnetization at the point of crossover from strong ferromagnetism to weak ferromagnetism and then another crossover from weak ferromagnetism to paramagnetism, it would be instructive to calculate the density of states $\rho_{\sigma}^{ \pm}(\omega)$ of hybridized bands, $\xi_{k \sigma}^{ \pm}$. It is given by

$$
\rho_{\sigma}^{ \pm}(\omega)=\sum_{k} \delta\left(\omega-\xi_{k \sigma}^{ \pm}\right)=\sum_{i} \delta\left(\omega-x_{i}\right)\left|\frac{\partial \xi_{k \sigma}^{ \pm}}{\partial \epsilon_{k}}\right|_{\epsilon_{k}=x_{i}}^{-1}
$$

where $x_{i}$ 's are the roots of $\omega-\xi_{k \sigma}^{ \pm}=0$. At a given energy $\omega$, the density of states is proportional to the slope of the hybridized bands at $\omega$. The crossover from strong to weak ferromagnetism is due to shift of the Fermi level from the upper hybridized up-spin band with larger density of states to the lower hybridized down-spin band with smaller density of states, resulting in a large decrease in the number of up-spin electrons. In the crossover from weak ferromagnetism to the paramagnetism, the change of the Fermi level in the lower hybridized up-spin band to the lower degenerate up and down spin bands acompanies a relatively weaker change of the density of states.

Figures 5 shows how the NN Coulomb interaction renormalizes the average occupation of the $f$-orbitals. In the absence of the NN Coulomb interaction the total magnetization, $m \approx n_{f \uparrow}$. In the strongly ferromagnetic regime $\left(G /\left|E_{f}\right|<2.0\right)$, for small values of $G$, up-spin electrons from the $f$-band are transferred to the conduction band through the hybridization interaction. With increasing values of $G$, more and more vacant sites are available and spin flip processes through hybridization interaction becomes energetically favourable. Therefore, we see an increase in the number of down-spin electrons and a decrease in the total magnetization. The weak ferromagnetic regime is stabilized by energy gain through the transfer of electrons from the $f$-band to the conduction band and by occupying next to nearest neighbour $f$-electron sites, since there are sufficiently large number of vacant sites available in this regime.

In this paper we have investigated the magnetic and non-magnetic ground states of the extended periodic Anderson model, using a variational method based on the one site approximation. We have shown through the calculation of the magnetic phase diagram that for $U=\infty$, the non-magnetic ground state is stabilized above a critical value of nearest neighbour Coulomb repulsion between the $f$-electrons. The one site approximation used in this paper gives exact calculation of the matrix elements in the limit of infinite dimensions. It will be very interesting to investigate the magnetic properties of the periodic Anderson model by including the (dimension) ${ }^{-1}$ contributions through two-site approximation 13 . Certainly, it is desirable to extend our calculations to study antiferromagnetic ground states also.

One of us (S.L) thanks Conselho Nacional de Desenvolvimento Cientifico e Technologico(CNPq), Brasil for financial assistance. 
${ }^{1}$ G. Aeppli and C. Broholm, Handbook on the Physics and Chemistry of Rare Earths, editors: K. A. Gschneider Jr., L. Eyring, G. H. Lander and G. R. Choppin vol. 19, (NorthHolland, Amsterdam, 1994), p. 123.

${ }^{2}$ N. Grewe and F. Steglich in Handbook on the Physics and Chemistry of Rare Earths, editors: K. A. Gschneider Jr. and L. Eyring, vol. 14, (North-Holland, Amsterdam, 1991), p. 343.

${ }^{3}$ H. R. Ott, Acta Physica Polonica A 85, 7 (1994).

${ }^{4}$ G. R. Stewart, Heavy-Fermion Systems, Rev. Mod. Phys. 56, 755 (1984).

${ }^{5}$ P. Fulde, Electron Correlations in Molecules and Solids (Springer, Berlin, 1991).

${ }^{6}$ P. A. Lee, T. M. Rice, J. W. Serene, L. J. Sham and J. W. Wilkins,, Comments in Solid State Physics 12, 99, (1986).

${ }^{7}$ N. Read and D. M. Newns, J. Phys. C 16, 3273 (1983).

${ }^{8}$ N. Read and D. M. Newns, J. Phys. C 16, L1055 (1983) .

${ }^{9}$ P. Coleman, Phys. Rev. B 29, 3035 (1984).

${ }^{10}$ A. M. Reynolds, D. M. Edwards and A. C. Hewson, J. Phys. Condensed Matter 4, 7589 (1992).

${ }^{11}$ K. Itai and P. Fazekas, cond-mat/9602056.

12 S. Lamba and S. K. Joshi, Phys. Rev.B 50, 8842 (1994).

${ }^{13}$ S. Lamba and S. K. Joshi, Phys. Rev. B 52, 7972, (1995).

${ }^{14}$ S. Lamba and S. K. Joshi, Physica B, 223 \& 224, 619, 1996.

${ }^{15}$ D Vollhardt in Proceeding of the International School of Physics Enrico Fermi Course CXXI, eds. R. A. Broglia and J. R. Schrieffer (North Holland, Amsterdam, 1994), p. 31. and references therein.

${ }^{16}$ M. C. Gutzwiller, Phys. Rev. Lett. 10, 159 (1963).

${ }^{17}$ D. Vollhardt, Rev. Mod. Phys. 56, 99 (1984).

${ }^{18}$ F. Gebhard, Phys. Rev. B 41, 9452 (1990).

${ }^{19}$ F. Gebhard, Phys. Rev. B 44, 992 (1991).

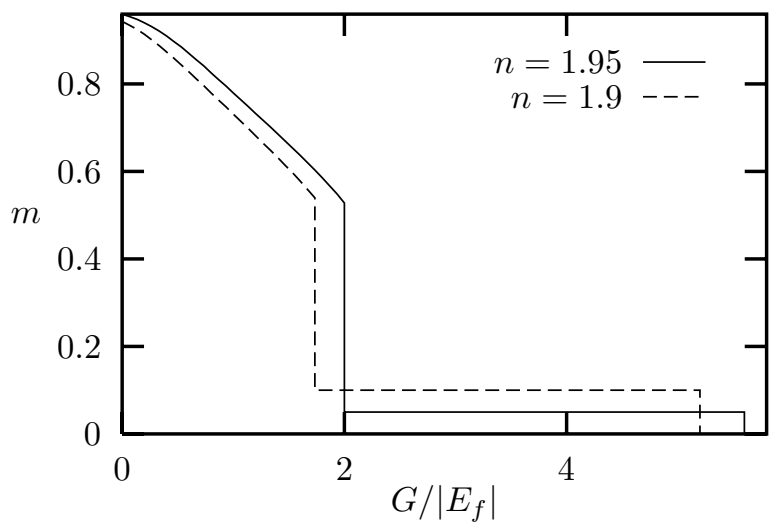

FIG. 2. The total magnetization as a function of the Coulomb repulsion between $f$-electrons on the nearest neighbour sites. Here we have taken the on-site Coulomb interaction to be infinitely large and bare hybridization, $V=1$.

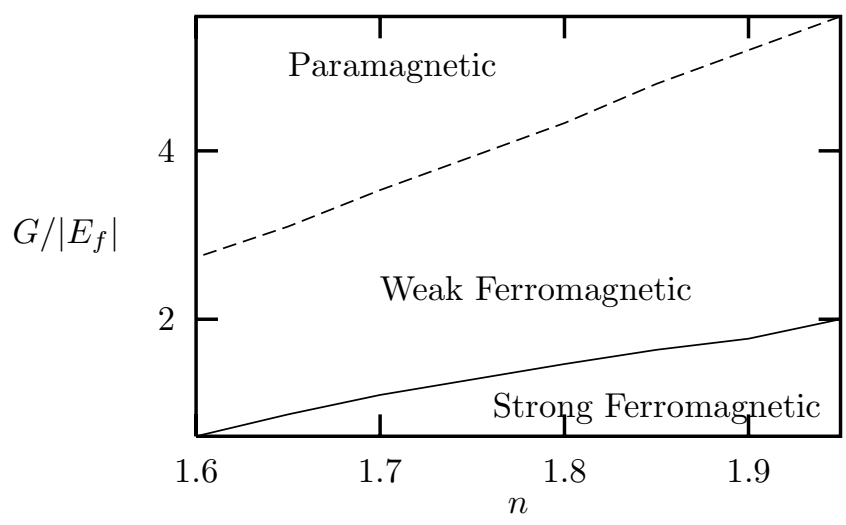

FIG. 3. Magnetic Phase diagram of extended periodic Anderson model 

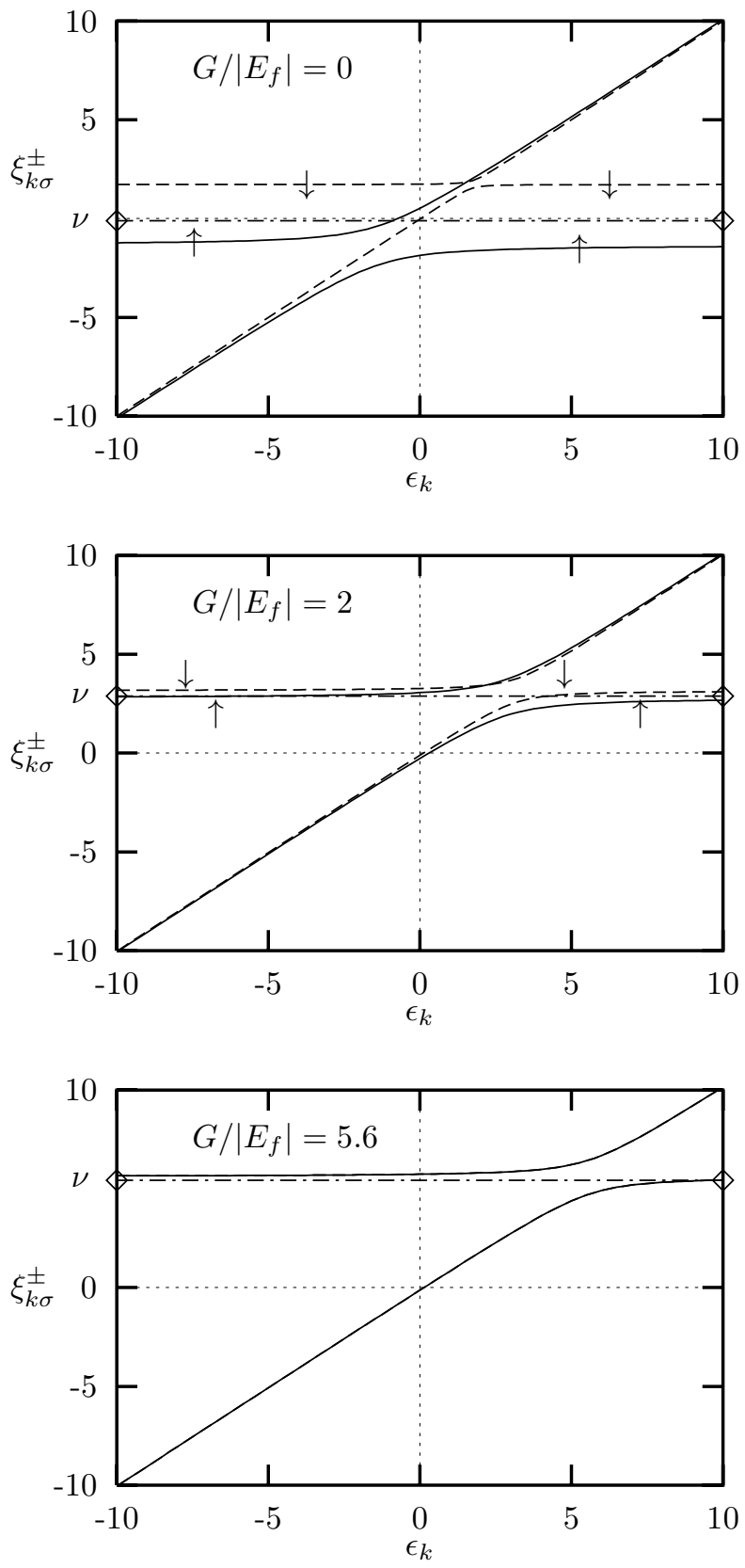

FIG. 4. Hybridized quasiparticle bands $\left(\xi_{k \sigma}^{ \pm}\right)$as a function of conduction band energy $\left(\epsilon_{k}\right)$ for the total density of electrons, $n=1.95$ and $V=1.0$. The $\uparrow$ and $\downarrow$ correspond to the hybridized up-spin band and hybridized down-spin band respectively. For $G /\left|E_{f}\right|=5.6$, the hybridized band are spin degenerate.

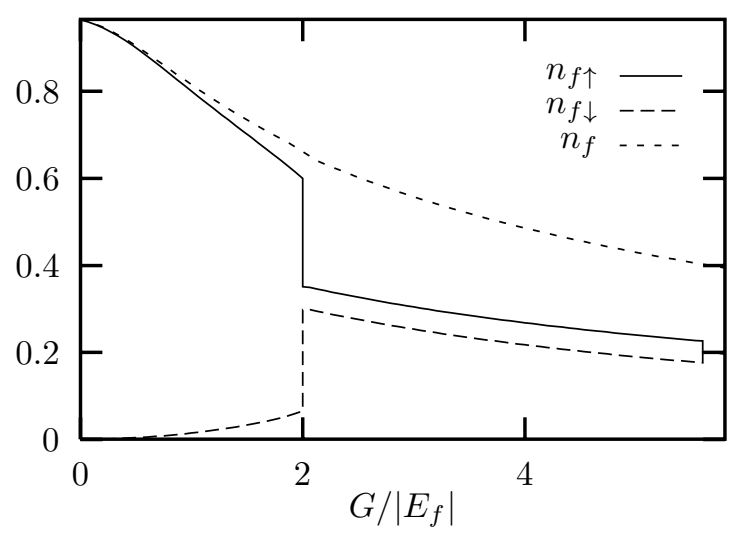

FIG. 5. The average occupation of the $f$-orbitals $n_{f \sigma}$ are plotted as a function of $G /\left|E_{f}\right|$ for $n=1.95$. 